\title{
Automatic Mammary Duct Detection in 3D Ultrasound
}

\author{
Mark J. Gooding ${ }^{1}$, Matthew Mellor ${ }^{2}$ Jacqueline A. Shipley ${ }^{1}$, \\ Kathy A. Broadbent ${ }^{3}$, and Dorothy A. Goddard ${ }^{4}$ \\ 1 Medical Physics Dept., Royal United Hospital, Bath, BA1 3NG, UK \\ mark.gooding@ruh-bath.swest.nhs.uk \\ ${ }^{2}$ Wolfson Medical Vision Laboratory, Dept. of Engineering Science, \\ University of Oxford, OX1 3PJ, UK \\ 3 Dept. of Medical Physics and Bioengineering, \\ Bristol General Hospital, Bristol, BS1 6SY, UK \\ 4 Breast Unit, Royal United Hospital, Bath, BA1 3NG, UK
}

\begin{abstract}
This paper presents a method for the initial detection of ductal structures within 3D ultrasound images using second-order shape measurements. The desire to detect ducts is motivated in a number of way, principally as step in the detection and assessment of ductal carcinoma in-situ. Detection is performed by measuring the variation of the local second-order shape from a prototype shape corresponding to a perfect tube. We believe this work is the first demonstration of the ability to detect sections of duct automatically in ultrasound images. The detection is performed with a view to employing vessel tracking method to delineate the full ductal structure.
\end{abstract}

\section{Introduction}

Most research into breast ultrasound analysis concerns the detection and diagnosis of lesions, therefore the detection of mammary ducts may seem somewhat peripheral. However, this is not the case; the role of the duct is central to the function of the breast, and consequently the ductal structures are an important area for analysis. Most malignancies originate in the epithelial tissues of the ducts, and it has been suggested that ductal echography, a process whereby the breast volume is systematically scanned along the line of duct, is the best way to manually find and diagnose lesions [1. The automatic detection of the ductal structure from 3D ultrasound could direct the reslicing and visualisation of the ultrasound data along the axis of each duct for clinical analysis, or enable automatic detection of anomalous ductal structure as early indicator of possible tumor development [2. The analysis of the duct may also have a role in the analysis of detected lesions, since the detection of duct-size features has been found to be an indicator of malignacy [3. To this end, comparison with the normal appearance of ducts in ultrasound images is required for diagnosis by ductal echography, although it should be noted that this appearance changes with age [41]. 
The extent of each lobe of the ductal system is also of importance. Typically, a carcinoma will spread along a duct, as ductal carcinoma in situ (DCIS), prior to any invasive spreading. As observed by Going and Moffat [5]:

If duct systems are independent of each other, then defining their boundaries prior to surgery for DCIS could assist in achieving complete removal of the affected 'lobe' while sparing the unaffected tissue.

Even in cases where the tumor is invasive, the ductal spread will be extensive and the lobe must be considered the minimum excision margin. However, the presence of any ductal anastomoses must be detected if the complete extent of possible spread is to be correctly assessed [6].

Once a ductal anomaly has been identified, ductal endoscopy and lavage provide possible routes for minimally invasive diagnosis. However, some lobular systems are not openly accessible on the nipple surface [5]. A complete tree of the ductal system would facilitate assessment of whether the duct can be accessed in such a way. Furthermore, a map of the 3D ductal structure would also facilitate registration based on anatomy [7, which would have application in assessing tumor changes following of treatment. Applications may also be found in assessment of problems associated with lactation [8], and in research into mammary development $[9]$.

Thus, the reasons motivating the automatic detection and segmention the ductal structure of the breast are:

- A method for the detection and diagnosis of lesions by comparison with normal ductal appearance.

- A Guide to excision margins by considering the possible intraductal spread.

- An aid for ductal endoscopy to assess access and navigation.

- Facilitation of anatomy-based registration for treatment assessment.

- An aid to the assessment and diagnosis of problems associated with lactation.

- Facilitation of mammary development research and other temporal studies of the breast.

In this paper, we review previous tube (mostly vascular) detection and tracking methods to motivate shape-based detection. A simple method for the initial detection of ductal structures is proposed and demonstrated. We believe this is the first demonstration of the ability to detect sections of duct automatically in ultrasound images. The paper concludes with a brief discussion of the direction of future research.

\section{Method}

\subsection{Previous Tube Detection and Tracking Approaches}

The detection of tubular structures has mostly been focused on the detection of vessels with MRA and CT and of bronchial tubes in CT. The approaches taken can be broadly divided into intensity-based segmentation and shape-based 
detection and tracking. Intensity-based detection (e.g. [10]) is not suitable for small ultrasound structures, such as ducts, since at a fine scale the image intensity is not directly representative of the tissue type. (At a coarse scale some tissue/intensity relationship may be noticed.) However, shape-based detection may show promise since the approximate structure of the tubes is still evident in the images, although significantly corrupted by speckle.

The second-order structure (the Hessian) of image intensity is often considered to be a good measure of the local shape. Frangi et al. 11 and Danielsson et al. 12 develop measures which describe the local 3D shape and "curvedness" based on the principal curvatures. Their work can be considered an extension of the shape index (SI), introduced by Koenderink and van Doorn [13], into 3D. The measures in [1214] are sufficient to uniquely describe the local second-order shape of an image. Subsequently, both authors develop further specific measures to detect vascular structure based on their local shape measures. Similarly, Sato et al. [15] use the principal curvatures of the image intensity to calculate measures of second-order structure corresponding to a sheets, tubes and spheres. These are used heuristically to segment image data from a range of modalities. All three authors demonstrate the detection of tube-like structure for the purpose of vessel segmentation in MRI volumes.

A number of effective methods have been developed for tracking of tubular structures. These can be roughly divided into those which track second-order structures (e.g. [1617]) and those based on medial node tracking (e.g. [1819]). The use of medial-nodes has be demonstrated for ultrasound data at a large scale 20, however the small size and poorly defined boundaries of ducts mean that this approach is not suited to the application of ductal tracking. Both of these approaches require initialisation. For the MRI and CT data, on which the methods have been demonstrated, the majority of initialisations have been achieved using an intensity threshold of the image data. However, intensity thresholding for the initial detection of ducts would be ineffective within ultrasound images. Section 2.2 addresses this initialisation problem, proposing a method for the initial detection of ducts within 3D ultrasound images, with a view to employing a tracking method to find the complete ductal structure.

\subsection{Simplified Detection Method}

In this section we propose a simple method for the detection of specific secondorder shape features directly from the principal curvatures, which could be used as an initialisation to the centerline tracking methods discussed previously. Although Danielsson's angular measures of shape provide a way to express the complete range of second-order shapes from volumes, the tubular shape measures introduced in [1121415] to detect vessels are unnecessarily complicated. We propose a simplified approach to tube detection. Any shape of interest within the second-order space can be uniquely expressed in terms of the three principal curvatures (the eigenvalues of the Hessian). Consequently, it is desirable to be able to measure the variation from a specific shape description expressed in terms of these curvatures. Therefore, we opt to detect tubular shapes directly from the 
principal curvatures, rather than introducing further measures of shape. The desired tube-shape prototype and the local shape can be expressed as vectors of the form

$$
\text { shape-vector }=\left(\begin{array}{c}
\kappa_{1} \\
\kappa_{2} \\
\kappa_{3}
\end{array}\right)
$$

where $\kappa_{1}, \kappa_{2}$ and $\kappa_{3}$ are the three principal curvatures. The orientation of this vector describes the local shape, while its length describes the degree of local curvedness. For a tubular shape the prototype is

$$
\text { tube }=\left(\begin{array}{c}
\frac{1}{\sqrt{2}} \\
\frac{1}{\sqrt{2}} \\
0
\end{array}\right) .
$$

We propose to measure the deviation of the local shape from the prototype shape as the normalised dot product between their shape vectors, as shown in equation 3. which can be thought of as an angular distance measure. This gives a result of 1 , for a perfect match, -1 for a perfect match of the opposite sign (i.e. same shape but opposite intensity structure), and zero where the local shape can be considered to be as far away as possible from the desired shape.

$$
\text { tubeness }=\left(\begin{array}{c}
\frac{\kappa_{1}}{\sqrt{\kappa_{1}+\kappa_{2}+\kappa_{3}}} \\
\frac{\kappa_{2}}{\sqrt{\kappa_{1}+\kappa_{2}+\kappa_{3}}} \\
\frac{\kappa_{3}}{\sqrt{\kappa_{1}+\kappa_{2}+\kappa_{3}}}
\end{array}\right) \cdot\left(\begin{array}{c}
\frac{1}{\sqrt{2}} \\
\frac{1}{\sqrt{2}} \\
0
\end{array}\right)=\cos \theta_{\text {tube }}
$$

Normalisation of the shape vectors ensures that this shape measure is invariant to contrast. Tubes are detected at multiple scales using scale normalised Gaussian convolution to generate progressively coarser scales [21]. The shape detected at a particular location may vary over the scales, as finer features are removed, e.g. A duct may be detected as tube like at a fine scale, but the more spherical shape of the local glandular tissue may be more prominent at a larger scale. Therefore, it is necessary to choose an appropriate scale to detect features of interest. We achieve this in a number of ways. First, we only process the scales over which we know the features exist, i.e. in looking for ducts we only search smaller scales. Second, we opt choose the scale at which each location looks most similar to the prototype shape vector. Detection of tubular features is achieved by accepting areas which do not deviate too far from the desired shape, and which have a high curvedness at the scale chosen. As noted in [12, curvedness, measured as $\sqrt{\sum \kappa_{i}^{2}}$ is not shape invariant; a spherical shape having higher curvedness than a tube-like one. However, we have chosen to detect features matching a single shape prototype, therefore using this measure is not a problem since we are comparing the curvedness of features with similar shapes.

\subsection{D Ultrasound Acquisition}

In-vivo acquisitions were carried out with consenting patients in accordance with an ethically approved protocol, using the radial scanning system described in 
[22 23], a Technos ultrasound scanner (Esaote S.p.A, Genova, Italy), and linear array probe (LA532 10-5) at 9MHz. The pathologies present were assessed by a clinician at the time of scanning, and subsequently by reviewing the full $3 \mathrm{D}$ data available. The $3 \mathrm{D}$ images were reconstructed on a $1 \mathrm{~mm}^{3}$ voxel array. The radial scanning system is appropriate for the detection of ducts for a number of reasons: ducts are oriented approximately radially from the nipple, therefore scanning radially allows sections of duct to be viewed along their length [1; the scanning system also uses low compression reducing the possibility that the ducts will collapse under the pressure of the probe during scanning; and the majority of the breast tissue volume is imaged, allowing the ductal structure to be viewed for most of the breast.

\section{Results}

For the examples given, features were detected by accepting voxels whose shape vectors were within $32^{\circ}$ of the prototype shape vector $\left(\cos \theta_{\text {tube }}=0.85\right)$. Empirically this was found to give good results, however the optimum distance has not been investigated. The curvedness used for feature acceptance was set for each $3 \mathrm{D}$ image as a percentage (typically in the range $40 \%$ to $60 \%$ ) of the maximum curvedness found for the tube feature over all scales. The exact value was found qualitatively in these examples, depending on the dynamic range and maximum curvedness of the particular scan. The scales used for detecting ducts and vessels had a Gaussian standard deviation ranging $\sigma=1$ to 3 voxels. This equivalent to a one standard deviation width of between 2 and $6 \mathrm{~mm}$, similar to the size of enlarged ducts 8. Detection was performed on a voxel-by-voxel basis.

Figures 1 and 2 demonstrate the potential of this system (both the radial scanner and detection method) to detect the ductal system in the breast. Figure

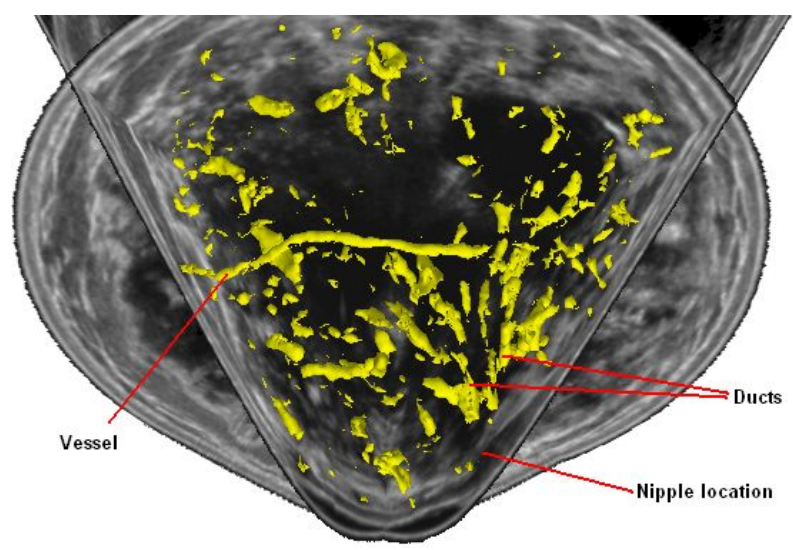

Fig. 1. The detection of ducts and vessels in a paracystic breast. Additional less interesting tube structures have been detected at cyst edges. However, these features still appear tube-like at the scale selected. 


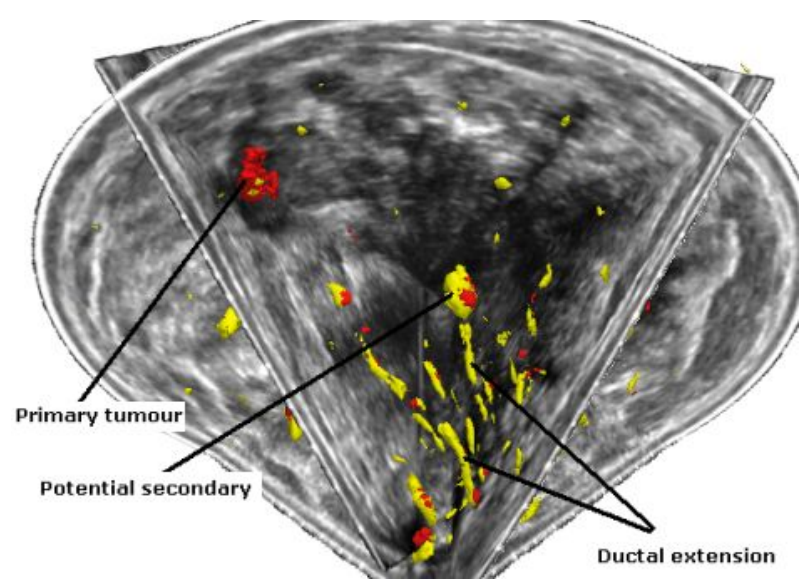

Fig. 2. The detection of ductal extension. A duct has been detected extending from the primary tumor. A possible secondary has also been detected within the same ductal tree, illustrating the importance of ductal tracking in assessing tumor invasiveness. Sphere-like structures (shown in red in the online proceedings) were also detected using a different shape prototype.

1 is a scan from a breast displaying polycystic changes and some ductal ectasia. Both vessels and ducts have been detected, although a number of less interesting detections have occurred at the edge of cysts. This is expected, since the edge of spherical objects appear tube-like in shape at small scales, and the cysts have high contrast resulting in high curvedness. Figure 2] shows the detection of ductal extension from a tumor in a breast containing a malignant ductal carcinoma. The extension of the tumor along the duct affects patient management, making mastectomy, rather than excision, the preferred option. The figure indicate areas which have been detected using a sphere-like structure prototype, rather than a tube-like one (shown in red in the online proceedings).

\section{Discussion, Conclusion and Future Work}

In this paper we have presented a method of tube-like feature detection for 3D ultrasound. Several methods of shape analysis and shape-based feature detection from the existing literature were considered. The method presented here simplifies these by detecting specific shapes directly from the principal curvatures, via comparison with a prototype feature, rather than introducing intermediate steps. The performance of this feature detector was demonstrated on 3D ultrasound data of breast volumes from two patients, showing the detection of ducts and vessels.

Several observations can be made from these results. First, that the detected objects correspond to perceptually significant image structures. However, interesting image structure does not necessarily equate to interesting pathology. This 
is not unexpected, since we are seeking a low-level shape feature rather than an anatomical feature. However, some knowledge-based constraints (e.g. we expect ducts to be long connected tubes) must be introduced to remove non-anatomical features for clinical use. Second, it is also noted that the shape of the detected volume does not correspond to the shape of the object itself. This is expected since a perfect tube will only be detected at its centre (see [18] for a discussion). Although the shape-measure itself cannot be used for accurate segmentation, it does give a pointer to scale and approximate tube position. Therefore, this detection method could be used as an initialisation for a centreline tracking method, such as in [16]17.

The challenge presented in 2 to provide a method for automatically detecting the entire ductal tree structure is significant. We believe this work is the first demonstration of the ability to detect sections of duct automatically in ultrasound images. Future work will consider the use of centerline tracking, by finding the connecting line which best corresponds to a tube as in [16, to form a continuous ductal tree structure. Quantitative evaluation of this tree structure will be performed by comparison with manual delineations. The extension of this ductal tree delineation to the normal breast, with non-enlarged ducts, is a long term goal.

\section{Acknowledgements}

We wish to acknowledge Francis Duck, Alison Noble, and Mike Halliwell for their help and advice in this work. Mark Gooding is supported by the research and development committee of the Royal United Hospital, Bath, NHS Trust.

\section{References}

1. Teboul, M., Halliwell, M.: Atlas of ultrasound and ductal echography of the breast. Blackwell Science Ltd, Oxford (1995)

2. Moskalik, A., Carson, P., Roubidoux, M.: 3D tracking and display of mammary ducts. In: Proc. IEEE Ultrasonics symposium 1995. Volume 1636. (1995) 11671170

3. Donohue, K., Huang, L., Genis, V., Forsberg, F.: Duct size detection and estimation in breast tissue. 1999 IEEE ultrasonics symposium (1999) 1353-1356

4. Nani, M., Piperno, G.: Il sistena duttale mammario. aspetti ecografici. Radiol Med 83 (1992) 745-750

5. Going, J., Moffat, D.: Escaping from flatland: clinical and biological aspects of human mammary duct anatomy in three dimensions. Journal of Pathology 203 (2004) 538-544

6. Ohtake, T., Kimijima, I., Fukushima, T., Yasuda, M., Sekikawa, K., Takenoshita, S., Abe, R.: Computer-assisted complete three-dimensional reconstruction of the mammary ductal/lobular systems. Cancer 19 (2001) 2263-2272

7. Aylward, S., Jomier, J., Weeks, S., Bullitt, E.: Registration and analysis of vascular images. Int. J. Comp. Vision 55 (2003) 123-138

8. Ramsay, D., Kent, J., Owens, R., Hartmann, P.: Ultrasound imaging of milk ejection in the breast of lactating women. Pediatrics 113 (2004) 361-367 
9. Moffat, D., Going, J.: Three dimensional anatomy of complete duct systems in human breast: pathological and developmental implications. Journal of Clinical Pathology 49 (1996) 48-52

10. Wilson, D., Noble, J.: An adaptive segmentation algorithm for time-of-flight mra data. IEEE Trans. Med. Imag. 18 (1999) 938-945

11. Frangi, A., Niessen, W., Vincken, K., Viergever, M.: Multiscale vessel enhancement filtering. MICCAI 1998, LNCS 1496 (1998) 130-137

12. Danielsson, P.E., Lin, Q., Ye, Q.Z.: Efficient detection of second-degree variations in 2D and 3D images. Journal of Visual Communication and Image Representation 12 (2001) 255-305

13. Koenderink, J., van Doorn, A.: Surface shape and curvature scales. Image Vision Comput. 10 (1992) 557-564

14. Lin, Q., Danielsson, P.E.: Stenosis detection using a new shape space for second order 3D-variations. IMPI 2001, LNCS 2082 (2001) 388-394

15. Sato, Y., Bhalerao, A., Nakajima, S., Shiraga, N., Tamura, S., Kikinis, R.: Tissue classification based on 3D local intensity structure for volume rendering. IEEE Transactions on visualization and computer graphics 6 (2000) 160-180

16. Aylward, S., Bullitt, E.: Initialization, noise, singularities and scale in hieght ridge traversal for tubular object centerline extraction. IEEE Trans. Med. Imag. 21 (2002) 61-75

17. Lorigo, L., Faugeras, O., Grimson, W., Keriven, R., Kikinis, R., Nabavi, A., Westin, C.: Curves: Curve evolution for vessel segmentation. Medical Image Analysis 5 (2001) 195-206

18. Krissian, K., Malandain, G., Ayache, N., Vaillant, R., Trousset, Y.: Model based detection of tubular structures in 3D images. Computer Vision and Image Understanding. 80 (2000) 130-171

19. Fridman, Y., Pizer, S., Aylward, S., Bullitt, E.: Segmenting 3D branching tubular structures using cores. MICCAI 2003, LNCS 2879 (2003) 570-577

20. Stetten, G., Pizer, S.: Medial-node models to identify and measure objects in real-time 3-D echocardiography. IEEE Trans. Med. Imag. 18 (1999) 1025-1034

21. ter Haar Romeny, B.: 6. In: Front-end vision and multi-scale image analysis. Kluwer Academic Publishers, Dordrecht (2003) 132-133

22. Shipley, J., Duck, F., Thomas, B.: Registration of ultrasound breast images acquired from a conical geometry. In: Proc Medical Image Understanding and Analysis 2003. (2003) 45-48

23. Shipley, J., Duck, F., Goddard, D., Hillman, M., Halliwell, M., Jones, M., Thomas, B.: Automated quantitative volumetric breast ultrasound data acquisition system. Ultrasound in Medicine and Biology 31 (2005) 905-917 